N. Obata

Nagoya Math. J.

Vol. 109 (1988), 91-107

\title{
A NOTE ON CERTAIN PERMUTATION GROUPS IN THE INFINITE DIMENSIONAL ROTATION GROUP
}

\author{
NOBUAKI OBATA
}

\section{Introduction}

In his book [8] P. Lévy discussed certain permutation groups of natural numbers in connection with the theory of functional analysis. Among them the group $\mathscr{G}$, called the Lévy group after T. Hida [3], has been studied along with Hida's theory of white noise analysis and has become very important keeping profound contact with the Lévy Laplacian which is an infinite dimensional analogue of the ordinary Laplacian ([6]).

This paper contains two topics on the Lévy group. We now give a summary of this paper.

I. Let $N$ be the set of all natural numbers. For an arbitrary subset $S$ of $N$, the upper density and the lower density of $S$ are defined by the formulae:

$$
\begin{aligned}
& \bar{\delta}(S)=\limsup _{N \rightarrow \infty} \frac{1}{N}|S \cap\{1,2, \cdots, N\}|, \\
& \underline{\delta}(S)=\liminf _{N \rightarrow \infty} \frac{1}{N}|S \cap\{1,2, \cdots, N\}|,
\end{aligned}
$$

respectively. If $\bar{\delta}(S)=\underline{\delta}(S)$, this quantity is called the density of $S$ and denoted by $\delta(S)$. We denote by $\mathscr{F}$ the family of all subsets which admit the density.

We denote by $\operatorname{Aut}(N)$ the group of all permutations of $N$. Let $\mathscr{G}(\delta)$ be the group of all permutations preserving density:

$$
\mathscr{G}(\delta)=\{g \in \operatorname{Aut}(N) ; g \mathscr{F}=\mathscr{F} \text { and } \delta(g(S))=\delta(S) \text { for any } S \in \mathscr{F}\}
$$

and let $\mathscr{G}_{0}$ be the group of all permutations whose supports are of null density:

Received July 3, 1986.

Revised December 27, 1986. 


$$
\mathscr{G}_{0}=\{g \in \operatorname{Aut}(N) ; \delta(\operatorname{supp} g)=0\} .
$$

For any $g \in \operatorname{Aut}(N)$ and $N \in N$, we put

$$
F_{N}(g)=\left\{n \in N ; \begin{array}{ll}
\text { (i) } 1 \leqslant n \leqslant N \text { and } g(n)>N ; & \text { or } \\
\text { (ii) } n>N \text { and } 1 \leqslant g(n) \leqslant N
\end{array}\right\} .
$$

Let $\mathscr{G}$ be the set of all permutations $g \in \operatorname{Aut}(N)$ satisfying

$$
F(g)=\limsup _{N \rightarrow \infty} \frac{1}{N}\left|F_{N}(g)\right|=0 .
$$

Then $\mathscr{G}$ becomes a subgroup of $\operatorname{Aut}(N)$ and is called the Lévy group. It will be proved that $\mathscr{G}_{\diamond} \subset \mathscr{G} \subset \mathscr{G}(\delta)$. A characterization of the Lévy group is given by

Theorem 1. For a permutation $g \in \operatorname{Aut}(N)$ the following three conditions are equivalent:

(i) $g \in \mathscr{G}$, i.e. $F(g)=0$;

(ii) $\bar{\delta}(g(S))=\bar{\delta}(S)$ for any $S \subset N$;

(iii) $\underline{\delta}(g(S))=\underline{\delta}(S)$ for any $S \subset N$.

In order to discuss the Lévy Laplacian we shall give another characterization of the Lévy group. Let $\ell^{\infty}$ be the Banach space of all bounded real sequences $a=\left(a_{n}\right)_{n=1}^{\infty}$ with the usual norm $\|a\|=\sup \left|a_{n}\right|$. Define two continuous functionals $L^{+}$and $L^{-}$on $\ell^{\infty}$ by the formulae:

$$
L^{+}(a)=\limsup _{N \rightarrow \infty} \frac{1}{N} \sum_{n=1}^{N} a_{n}, \quad L^{-}(a)=\liminf _{N \rightarrow \infty} \frac{1}{N} \sum_{n=1}^{N} a_{n},
$$

respectively. The group Aut $(N)$ acts on $\ell^{\infty}$ as coordinate permutations, i.e. by means of the maps:

$$
a \longmapsto g a=\left(a_{g-1(n)}\right)_{n=1}^{\infty}, \quad a=\left(a_{n}\right)_{n=1}^{\infty} \in \ell^{\infty}, \quad g \in \operatorname{Aut}(N) .
$$

With these notations we may prove

Theorem 2. For a permutation $g \in \operatorname{Aut}(N)$ the following three conditions are equivalent:

(i) $g \in \mathscr{G}$, i.e. $F(g)=0$;

(ii) $L^{+}(g a)=L^{+}(a)$ for any $a \in \ell^{\infty}$;

(iii) $L^{-}(g a)=L^{-}(a)$ for any $a \in \ell^{\infty}$.

II. Let $\mathscr{D}$ be the subspace of all sequences $a \in \ell^{\infty}$ such that $L^{+}(a)=$ $L^{-}(a)$ and put 


$$
L(a)=\lim _{N \rightarrow \infty} \frac{1}{N} \sum_{n=1}^{N} a_{n}, \quad a=\left(a_{n}\right)_{n=1}^{\infty} \in \mathscr{D} .
$$

Then $\mathscr{D}$ is a closed subspace of $\ell^{\infty}$ and $L$ a continuous linear functional on it. Define the group $\mathscr{G}(L, \mathscr{D})$ by

$$
\mathscr{G}(L, \mathscr{D})=\{g \in \operatorname{Aut}(N) ; g \mathscr{D}=\mathscr{D} \text { and } L(g a)=L(a) \text { for any } a \in \mathscr{D}\} .
$$

It is noted that the Lévy group $\mathscr{G}$ is a proper subgroup of $\mathscr{G}(L, \mathscr{D})$.

We then come to the Lévy Laplacian. Let $E=C^{\infty}\left(S^{1}\right)$ and $H=$ $L^{2}\left(S^{1}\right)$ be the nuclear space of all $R$-valued $C^{\infty}$-functions on the circle $S^{1}$ and the Hilbert space of all $\boldsymbol{R}$-valued square integrable functions, respectively. Once we fix a complete orthonormal system $\left\{e_{n}\right\}_{n=1}^{\infty} \subset E$ for $H$, the group Aut $(N)$ may be regarded as a subgroup of the orthogonal group $O(H)$ in an obvious manner.

Let $\operatorname{Dom}_{H}(\Delta)$ (resp. $\operatorname{Dom}_{E}(\Delta)$ ) be the space of all $R$-valued continuous functions on $H$ (resp. $E$ ) satisfying the following two conditions:

(i) $\sup _{n}\left|\frac{\partial^{2} f}{\partial \xi_{n}^{2}}(\xi)\right|<\infty$ for any $\xi \in H$ (resp. $E$ );

(ii) $\lim _{N \rightarrow \infty} \frac{1}{N} \sum_{n=1}^{N} \frac{\partial^{2} f}{\partial \xi_{n}^{2}}(\xi)$ exists for any $\xi \in H$ (resp. $E$ ).

Here we note that every $R$-valued function $f$ on $H$ (resp. $E$ ) is regarded as one of countably infinite variables $\left(\xi_{n}\right)_{n=1}^{\infty}$ through the Fourier series expansion $\xi=\sum \xi_{n} e_{n}, \xi \in H$ (resp. $\left.\xi \in E\right)$. For $f \in \operatorname{Dom}_{H}(\Delta)\left(\operatorname{resp} . \operatorname{Dom}_{E}(\Delta)\right)$, we put

$$
(\Delta f)(\xi)=\lim _{N \rightarrow \infty} \frac{1}{N} \sum_{n=1}^{N} \frac{\partial^{2} f}{\partial \xi_{n}^{2}}(\xi), \quad \xi \in H(\text { resp. } E) .
$$

The linear operator $\Delta$ is called the Lévy Laplacian. In some literature the Lévy Laplacian is denoted by $\Delta_{L}$ in order to avoid confusing it with the number operator (or the Ornstein-Uhlenbeck operator). In this paper, however, it is denoted by $\Delta$ for simplicity.

For each $g \in O(H)$ we define an operator $U(g)$ by

$$
(U(g) f)(\xi)=f\left(g^{-1} \xi\right), \quad \xi \in H,
$$

where $f$ is an arbitrary function on $H$. For a function on $E$ we need further notation, since the space $E$ is not stable under $O(H)$. Following H. Yoshizawa [11] we call an orthogonal operator $g \in O(H)$ a rotation of $E$ if its restriction to $E$ induces a topological automorphism of $E$. Let 
$O(E, H)$ denote the group of all rotations of $E$. It plays an important role in probability theory ([4], [5], [10], etc). Restricted to the group $O(E, H)$, the above formula defines an operator $U(g)$ acting on the space of functions on $E$. We are now in a position to state the main results on the Lévy Laplacian.

TheOREM 3. The group $\mathscr{G}(L, \mathscr{D})$ consists of all permutations which commute with the Lévy Laplacian $\Delta$ with the domain $\operatorname{Dom}_{H}(\Delta)$.

TheOrem 4. The group $\mathscr{G}(L, \mathscr{D}) \cap O(E, H)$ consists of all permutations which commute with the Lévy Laplacian $\Delta$ with the domain $\operatorname{Dom}_{E}(\Delta)$.

From the above results it follows that the Lévy group $\mathscr{G}$ (resp. the group $\mathscr{G} \cap O(E, H)$ ) commutes with the Lévy Laplacian $\Delta$ with the domain $\operatorname{Dom}_{H}(\Delta)\left(\right.$ resp. $\left.\operatorname{Dom}_{E}(\Delta)\right)$. This fact was first remarked by T. Hida [3].

ACKNowledgement. I would like to express my gratitude to the referee for his suggestions and to Professor T. Hida for his valuable advice and constant encouragement.

\section{$\S 1$. Density of natural numbers and the Lévy group}

Let $N$ denote the set of all natural numbers. For an arbitrary subset $S \subset N$ we put

$$
\begin{aligned}
& \bar{\delta}(S)=\limsup _{N \rightarrow \infty} \frac{1}{N}|S \cap\{1,2, \cdots, N\}|, \\
& \underline{\delta}(S)=\liminf _{N \rightarrow \infty} \frac{1}{N}|S \cap\{1,2, \cdots, N\}|,
\end{aligned}
$$

where $|\cdot|$ denotes the cardinality. The quantity $\bar{\delta}(S)(\operatorname{resp} . \underline{\delta}(S))$ is called the upper density (resp. lower density) of $S$. If $\bar{\delta}(S)=\underline{\delta}(S)$, this is called the density of $S$ and denoted by $\delta(S)$. We denote by $\mathscr{F}$ the collection of all subsets of $N$ which admit the density. Then the triple $(N, \mathscr{F}, \delta)$ is analoguous to a probability space but not quite. In fact, $\mathscr{F}$ is not finitely additive. Nevertheless, we have the following

Proposition 1.1.

(1) If $S \in \mathscr{F}$, then $S^{c} \in \mathscr{F}$ and $\delta\left(S^{c}\right)=1-\delta(S)$.

(2) If $S_{1} \in \mathscr{F}$ and $S_{2} \in \mathscr{F}$, then $\bar{\delta}\left(S_{1} \cup S_{2}\right) \leqslant \delta\left(S_{1}\right)+\delta\left(S_{2}\right)$.

(3) Let $S_{1} \in \mathscr{F}$ and $S_{2} \in \mathscr{F}$. If $\delta\left(S_{1} \cap S_{2}\right)=0$, then $S_{1} \cup S_{2} \in \mathscr{F}$ and $\delta\left(S_{1} \cup S_{2}\right)=\delta\left(S_{1}\right)+\delta\left(S_{2}\right)$. 
The proof is easy and omitted. Let $\operatorname{Aut}(N)$ be the group of all permutations of $N$ and $\mathscr{G}(\delta)$ the subgroup of all permutations which preserve the density:

$$
\mathscr{G}(\delta)=\{g \in \operatorname{Aut}(N) ; g \mathscr{F}=\mathscr{F} \text { and } \delta(g(S))=\delta(S) \text { for any } S \in \mathscr{F}\} .
$$

For any $g \in \operatorname{Aut}(N)$ we put

$$
\operatorname{supp} g=\{n \in N ; g(n) \neq n\} .
$$

Then $g$ is a bijection from supp $g$ onto itself. In particular, supp $g=$ $\operatorname{supp} g^{-1}$. By Proposition 1.1 (2) the set

$$
\mathscr{G}_{0}=\{g \in \operatorname{Aut}(N) ; \delta(\operatorname{supp} g)=0\}
$$

forms a subgroup of $\operatorname{Aut}(N)$. It is known that the group $\mathscr{G}_{0}$ is closely related to the average power introduced in [1], however, we shall not go into a detailed discussion in this paper.

For $g \in \operatorname{Aut}(N)$ we put

$$
\begin{aligned}
& F_{N}^{+}(g)=\{n \in N ; 1 \leqslant n \leqslant N, g(n)>N\}, \\
& F_{N}^{-}(g)=\{n \in N ; n>N, 1 \leqslant g(n) \leqslant N\}
\end{aligned}
$$

and

$$
F_{N}(g)=F_{N}^{+}(g) \cup F_{N}^{-}(g) \quad \text { (disjoint union) } .
$$

Obviously, $\left|F_{N}(g)\right|=2\left|F_{N}^{+}(g)\right|=2\left|F_{N}^{-}(g)\right|$. Define a function on Aut $(N)$ by

$$
F(g)=\limsup _{N \rightarrow \infty} \frac{1}{N}\left|F_{N}(g)\right|, \quad g \in \operatorname{Aut}(N),
$$

and put

$$
\mathscr{G}=\{g \in \operatorname{Aut}(N) ; F(g)=0\} .
$$

As is easily seen, $\mathscr{G}$ forms a subgroup of $\operatorname{Aut}(N)$ and is called the Lévy group after T. Hida. It was first introduced by P. Lévy in connection with equally dense complete orthonormal systems ([8]).

Proposition 1.2. $\mathscr{G}_{0} \subset \mathscr{G} \subset \mathscr{G}(\delta)$.

Proof. From the inequality

$$
F(g) \leqslant 2 \bar{\delta}(\operatorname{supp} g), \quad g \in \operatorname{Aut}(N),
$$

which is easily seen, the inclusion $\mathscr{G}_{0} \subset \mathscr{G}$ follows. We shall prove the inclusion $\mathscr{G} \subset \mathscr{G}(\delta)$. First note that, for any $g \in \operatorname{Aut}(N)$ and $S \subset N$ 


$$
S \cap\{g(1), \cdots, g(N)\} \subset(S \cap\{1, \cdots, N\}) \cup F_{N}^{+}(g) .
$$

Therefore, if $g \in \mathscr{G}$, we have

$$
\begin{aligned}
\bar{\delta}\left(g^{-1}(S)\right) & =\limsup _{N \rightarrow \infty} \frac{1}{N}|S \cap\{g(1), \cdots, g(N)\}| \\
& \leqslant \limsup _{N \rightarrow \infty}\left(\frac{1}{N}|S \cap\{1, \cdots, N\}|+\frac{1}{N}\left|F_{N}^{+}(g)\right|\right)=\bar{\delta}(S) .
\end{aligned}
$$

Since $\mathscr{G}$ is a group, we see that

$$
\bar{\delta}(g(S))=\bar{\delta}(S) \text { for any } S \subset N \text { and } g \in \mathscr{G} .
$$

Similarly,

$$
\underline{\delta}(g(S))=\underline{\delta}(S) \text { for any } S \subset N \text { and } g \in \mathscr{G} .
$$

Hence $\mathscr{G} \subset \mathscr{G}(\delta)$ as desired.

The following result was established during the above proof.

CoRollary 1.3. The upper density $\bar{\delta}$ and the lower density $\underline{\delta}$ are invariant under the Lévy group.

ExAmple 1.4 ([3]). Let $0=N_{0}<N_{1}<\ldots$ be an increasing sequence of integers. For any permutation $g \in \operatorname{Aut}(N)$ which leave every subset $\left\{N_{k-1}+1, \cdots, N_{k}\right\}$ invariant, we have

$$
F(g) \leq 2 \limsup _{k \rightarrow \infty}\left(\frac{N_{k}}{N_{k-1}}-1\right)
$$

Therefore, if $\lim _{k \rightarrow \infty} N_{k} / N_{k-1}=1, g$ belongs to $\mathscr{G}$. In particular, $\mathscr{G}_{0}$ is a proper subgroup of $\mathscr{G}$.

\section{§2. Characterizations of the Lévy group}

We showed in the previous section that the upper density $\bar{\delta}$ and the lower density $\underline{\delta}$ are both invariant under the Lévy group $\mathscr{G}$. As we see below, this property actually characterizes the Lévy group.

Theorem 1. For a permutation $g \in \operatorname{Aut}(N)$ the following three conditions are equivalent:

(i ) $g \in \mathscr{G}$, i.e. $F(g)=0$;

(ii) $\bar{\delta}(g(S))=\bar{\delta}(S)$ for any $S \subset N$;

(iii) $\underline{\delta}(g(S))=\underline{\delta}(S)$ for any $S \subset N$. 
The following result whose proof is elementary and omitted will be used in the proof of Theorem 1 .

Lemma 2.1. Let $0=N_{0}<N_{1}<\cdots$ be an increasing sequence of integers and let $\left\{p_{n}\right\}_{n=1}^{\infty}$ be a sequence of integers satisfying $0 \leqslant p_{n} \leqslant N_{n}-N_{n-1}$. For any subset $S \subset N$ satisfying

$$
\left|S \cap\left\{N_{n-1}+1, N_{n-1}+2, \cdots, N_{n}\right\}\right|=p_{n}, \quad n \geqslant 1,
$$

we have

(1) $\bar{\delta}(S) \leqslant \limsup _{n \rightarrow \infty} \frac{1}{N_{n-1}+p_{n}} \sum_{k=1}^{n} p_{k}$;

(2) $\delta(S) \geqslant \liminf _{n \rightarrow \infty} \frac{1}{N_{n}-p_{n}} \sum_{k=1}^{n-1} p_{k}$.

Proof of Theorem 1. The implication (i) $\Rightarrow$ (ii) and (iii) has been already shown in Corollary 1.3.

Suppose that a permutation $g$ does not belong to $\mathscr{G}$. It suffices to construct a subset $S \subset N$ such that $\bar{\delta}(g(S))<\bar{\delta}(S)$ and $\underline{\delta}(g(S))<\underline{\delta}(S)$. By assumption, there exists a sequence $0=N_{0}<N_{1}<N_{2}<\cdots$ such that

$$
\lim _{n \rightarrow \infty} \frac{1}{N_{n}}\left|F_{N_{n}}^{+}(g)\right|=\frac{1}{2} F(g)>0 .
$$

For simplicity we put $\left|F_{N_{n}}^{+}(g)\right|=p_{n}$. Here we may assume that $g(1), \cdots$, $g\left(N_{n}\right)<N_{n+1}$. Put

$$
S=\bigcup_{n=1}^{\infty} F_{N_{n}}^{+}(g)
$$

Then we obtain

$$
\begin{aligned}
& \left|S \cap\left\{N_{n-1}+1, \cdots, N_{n}\right\}\right|=p_{n}, \\
& \left|g(S) \cap\left\{N_{n-1}+1, \cdots, N_{n}\right\}\right|=p_{n-1} .
\end{aligned}
$$

Given $\varepsilon>0$, there exists $n_{0} \geqslant 1$ such that

$$
\left(\frac{1}{2} F(g)-\varepsilon\right) N_{n} \leqslant p_{n} \leqslant\left(\frac{1}{2} F(g)+\varepsilon\right) N_{n} \quad \text { for all } n>n_{0} .
$$

Then, for any $n>n_{0}$ we have

$$
\begin{aligned}
\mid S \cap & \left\{1, \cdots, N_{n}\right\} \mid=\sum_{k=1}^{n} p_{k} \\
& \geqslant\left(\frac{1}{2} F(g) \quad \varepsilon\right) \sum_{k=n_{0}+1}^{n} N_{k}+\sum_{k=1}^{n_{0}} p_{k} .
\end{aligned}
$$


Hence,

$$
\begin{aligned}
\bar{\delta}(S) & \geqslant \limsup _{n \rightarrow \infty} \frac{1}{N_{n}}\left|S \cap\left\{1, \cdots, N_{n}\right\}\right| \\
& \geqslant\left(\frac{1}{2} F(g)-\varepsilon\right) \limsup _{n \rightarrow \infty} \frac{1}{N_{n}}\left(\sum_{k=n_{0}+1}^{n} N_{k}\right) .
\end{aligned}
$$

On the other hand, it follows from Lemma 2.1 that

$$
\begin{aligned}
\bar{\delta}(g(S)) & \leqslant \limsup _{n \rightarrow \infty} \frac{1}{N_{n}+p_{n}} \sum_{k=1}^{n} p_{k} \\
& \leqslant\left(\frac{1}{2} F(g)+\varepsilon\right) \limsup _{n \rightarrow \infty} \frac{1}{N_{n}+p_{n}} \sum_{k=n_{0}+1}^{n} N_{k} \\
& =\left(\frac{1}{2} F(g)+\varepsilon\right)\left(1+\frac{1}{2} F(g)\right)^{-1} \limsup _{n \rightarrow \infty} \frac{1}{N_{n}} \sum_{k=n_{0}+1}^{n} N_{k} .
\end{aligned}
$$

Take $\varepsilon>0$ sufficiently small such that

$$
\frac{1}{2} F(g)-\varepsilon>\left(\frac{1}{2} F(g)+\varepsilon\right)\left(1+\frac{1}{2} F(g)\right)^{-1} .
$$

Viewing (2.1) and (2.2), we have $\bar{\delta}(S)>\bar{\delta}(g(S))$. In a similar manner we can show that $\underline{\delta}(S)>\underline{\delta}(g(S))$.

Q.E.D.

In the rest of this section we give another characterization of the Lévy group. Let $\ell^{\infty}$ be the Banach space of all bounded real sequences $a=\left(a_{n}\right)_{n=1}^{\infty}$ with the norm $\|a\|=\sup \left|a_{n}\right|$. The group $\operatorname{Aut}(N)$ acts on $\ell^{\infty}$ as coordinate permutations, namely, by means of the maps:

$$
a=\left(a_{n}\right)_{n=1}^{\infty} \longmapsto g a=\left(a_{g-1(n)}\right)_{n=1}^{\infty} .
$$

We now introduce two functionals $L^{+}$and $L^{-}$on $\ell^{\infty}$ :

$$
L^{+}(a)=\limsup _{N \rightarrow \infty} \frac{1}{N} \sum_{n=1}^{N} a_{n}, \quad L^{-}(a)=\liminf _{N \rightarrow \infty} \frac{1}{N} \sum_{n=1}^{N} a_{n}
$$

where $a=\left(a_{n}\right)_{n=1}^{\infty} \in \ell^{\infty}$. Then one can prove the following assertion easily.

\section{LEMMA 2.2 .}

(1) $L^{+}(-a)=-L^{-}(a), a \in \ell^{\infty}$.

(2) $\liminf _{n \rightarrow \infty} a_{n} \leqslant L^{+}(a) \leqslant \limsup _{n \rightarrow \infty} a_{n}, a=\left(a_{n}\right)_{n=1}^{\infty} \in \ell^{\infty}$.

(3) $\left|L^{+}(a)-L^{+}(b)\right| \leqslant\|a-b\|, a, b \in \ell^{\infty}$.

The quantities $\lim \inf a_{n}$ and $\lim \sup a_{n}($ see Lemma $2.2(2))$ are invariant under the action of $\operatorname{Aut}(N)$, namely, for any $g \in \operatorname{Aut}(N)$, 


$$
\liminf _{n \rightarrow \infty} a_{g^{-1(n)}}=\liminf _{n \rightarrow \infty} a_{n} \text { and } \limsup _{n \rightarrow \infty} a_{g^{-1(n)}}=\limsup _{n \rightarrow \infty} a_{n} .
$$

While, $L^{+}(g a)$ varies when $g$ runs over Aut $(N)$. More precisely, we have the following result whose proof is easy.

Proposition 2.3. Let $a \in \ell^{\infty}$ be fixed. Then the range of the map $g \mapsto L^{+}(g a), g \in \operatorname{Aut}(N)$, coincides with the closed interval [liminf $a_{n}$, $\left.\lim \sup a_{n}\right]$.

Thus we are interested in the permutation group which keeps the functionals $L^{+}$and $L^{-}$invariant. In this connection we have

Theorem 2. For a permutation $g \in \operatorname{Aut}(N)$ the following three conditions are equivalent:

(i) $g \in \mathscr{G}$, i.e. $F(g)=0$;

(ii) $L^{+}(g a)=L^{+}(a)$ for any $a \in \ell^{\infty}$;

(iii) $L^{-}(g a)=L^{-}(a)$ for any $a \in \ell^{\infty}$.

This is an immediate consequence of the following

Lemma 2.4. For a permutation $g \in \operatorname{Aut}(N)$, the following three conditions are equivalent:

(i) $g \in \mathscr{G}$, i.e. $F(g)=0$;

(ii) $\lim _{N \rightarrow \infty} \frac{1}{N} \sum_{n=1}^{N}\left(a_{n}-a_{g(n)}\right)=0$ for all $a \in \ell^{\infty}$;

(iii) $\limsup _{N \rightarrow \infty} \frac{1}{N} \sum_{n=1}^{N} a_{n}=\limsup _{N \rightarrow \infty} \frac{1}{N} \sum_{n=1}^{N} a_{g(n)}$ for all $a \in \ell^{\infty}$.

Proof. The implication (i) $\Rightarrow$ (ii) is easily shown with the help of the inequality:

$$
\left|\sum_{n=1}^{N}\left(a_{n}-a_{g(n)}\right)\right| \leqslant\left|F_{N}(g)\right|\|a\|, \quad a \in \ell^{\infty} .
$$

Next we show the implication (ii) $\Rightarrow$ (iii). Let $a \in \ell^{\infty}$. Then

$$
\begin{aligned}
\limsup _{N \rightarrow \infty} & \frac{1}{N} \sum_{n=1}^{N} a_{g(n)} \\
& \leqslant \limsup _{N \rightarrow \infty} \frac{1}{N} \sum_{n=1}^{N}\left(a_{g(n)}-a_{n}\right)+\limsup _{N \rightarrow \infty} \frac{1}{N} \sum_{n=1}^{N} a_{n} \\
& =\limsup _{N \rightarrow \infty} \frac{1}{N} \sum_{n=1}^{N} a_{n} .
\end{aligned}
$$

Since the set of all permutations $g \in \operatorname{Aut}(N)$ satisfying the condition (ii) 
forms a subgroup, we have (iii).

Finally we show that (iii) implies (i). Suppose that $g$ does not belong to $\mathscr{G}$. It follows from Theorem 1 that there exists a subset $S \subset N$ such that $\bar{\delta}(g(S)) \neq \bar{\delta}(S)$. We define $a=\left(a_{n}\right)_{n=1}^{\infty} \in l^{\infty}$ by

$$
a_{n}=1 \text { if } n \in S ;=0 \text { otherwise. }
$$

Since

$$
\bar{\delta}(S)=\limsup _{N \rightarrow \infty} \frac{1}{N} \sum_{n=1}^{N} a_{n}
$$

and

$$
\bar{\delta}(g(S))=\limsup _{N \rightarrow \infty} \frac{1}{N} \sum_{n=1}^{N} a_{g-1(n)},
$$

(iii) does not hold. This completes the proof.

Q.E.D.

\section{§3. The Lévy Laplacian}

Let $\mathscr{D}$ be the subspace of all bounded sequences $a \in \ell^{\infty}$ such that $L^{+}(a)=L^{-}(a)$. Then $\mathscr{D}$ is a closed subspace of $\ell^{\infty}$ and the functional

$$
L(a)=\lim _{N \rightarrow \infty} \frac{1}{N} \sum_{n=1}^{N} a_{n}, \quad a=\left(a_{n}\right)_{n=1}^{\infty} \in \mathscr{D},
$$

is continuous and linear. We now define a subgroup $\mathscr{G}(L, \mathscr{D})$ of $\operatorname{Aut}(N)$ :

$$
\mathscr{G}(L, \mathscr{D})=\{g \in \operatorname{Aut}(N) ; g \mathscr{D}=\mathscr{D} \text { and } L(g a)=L(a) \text { for any } a \in \mathscr{D}\} .
$$

Then we have the following

Proposition 3.1. $\mathscr{G} \subset \mathscr{G}(L, \mathscr{D}) \subset \mathscr{G}(\delta)$.

Proof. The inclusion $\mathscr{G} \subset \mathscr{G}(L, \mathscr{D})$ follows from Theorem 2. The inclusion $\mathscr{G}(L, \mathscr{D}) \subset \mathscr{G}(\delta)$ is immediate.

Q.E.D.

Let $H$ be a real Hilbert space with a complete orthonormal system $\left\{e_{n}\right\}_{n=1}^{\infty}$. Each $\xi \in H$ is sometimes identified with a real sequence $\left(\xi_{n}\right)_{n=1}^{\infty}$ through the Fourier series expansion. Let $O(H)$ be the group of all orthogonal operators on $H$. With each $g \in \operatorname{Aut}(N)$ we associate an orthogonal operator $g$ (denoted by the same symbol) by means of the map:

$$
\xi \longmapsto g \xi=\left(\xi_{g-1(n)}\right)_{n=1}^{\infty}, \quad \xi=\left(\xi_{n}\right)_{n=1}^{\infty} \in H .
$$

Thus, we may regard Aut $(N)$ as a subgroup of $O(H)$. 
Let $\operatorname{Dom}_{H}(\Delta)$ be the space of all $R$-valued continuous functions on $H$ satisfying the following two conditions:

(i) $\sup _{n}\left|\frac{\partial^{2} f}{\partial \xi_{n}^{2}}(\xi)\right|<\infty$ for all $\xi \in H$;

(ii) $\lim _{N \rightarrow \infty} \frac{1}{N} \sum_{n=1}^{N} \frac{\partial^{2} f}{\partial \xi_{n}^{2}}(\xi)$ exists for all $\xi \in H$.

Here we note that every function $f(\xi)$ on $H$ is regarded as one of infinitely many real variables $\left(\xi_{n}\right)_{n=1}^{\infty}$. If $f \in \operatorname{Dom}_{H}(\Delta)$, we put

$$
(\Delta f)(\xi)=\lim _{N \rightarrow \infty} \frac{1}{N} \sum_{n=1}^{N} \frac{\partial^{2} f}{\partial \xi_{n}^{2}}(\xi), \quad \xi \in H .
$$

The linear operator $\Delta$ is called the Lévy Laplacian.

ExAmple 3.2. A function $f(\xi)$ is called a monomial of degree $p$ if it admits the expression:

$$
f(\xi)=\left(\xi^{\otimes p}, a\right), \quad a \in S^{p} H(p \text {-th symmetric tensor product) } .
$$

Every monomial belongs to $\operatorname{Dom}_{H}(\Delta)$ and $(\Delta f)(\xi)=0$.

Example 3.3. Consider a function $f(\xi)$ given by

$$
f(\xi)=\left(A \xi^{\otimes p}, \xi^{\otimes p}\right), \quad A \in \boldsymbol{B}\left(S^{p} H\right) .
$$

If the limit

$$
2 p^{2} \lim _{N=\infty} \frac{1}{N} \sum_{n=1}^{N}\left(A\left(e_{n} \otimes \xi^{\otimes(p-1)}\right), e_{n} \otimes \xi^{\otimes(p-1)}\right)
$$

exists for any $\xi \in H$, the function $f$ belongs to $\operatorname{Dom}_{H}(\Delta)$ and $(\Delta f)(\xi)$ is equal to the limit (3.4). If $p=1$, any function of the form (3.3) is called a quadratic functional on $H$.

From now on, we assume that $H$ is the Hilbert space of all $R$-valued square integrable functions on the circle. Each $\xi \in H$ is regarded as a function on $\boldsymbol{R}$ with period 1. We fix a complete orthonormal system $\left\{e_{n}\right\}_{n=1}^{\infty}$ as follows:

$$
e_{1}(t) \equiv 1, \quad e_{2 n}(t)=\sqrt{2} \sin 2 \pi n t, \quad e_{2 n+1}(t)=\sqrt{2} \cos 2 \pi n t,
$$

where $n \geqslant 1$. This system is equally dense ([8]). The Lévy Laplacian is closely related with the functional derivatives of second order through an equally dense complete orthonormal system ([6]). 
EXAMPLE 3.4 ([2], [6]). Let $a(t)$ be an essentially bounded function on $S^{1}$. Then the function

$$
f(\xi)=\int_{0}^{1} a(t) \xi(t)^{2} d t, \quad \xi \in H
$$

belongs to $\operatorname{Dom}_{H}(\Delta)$ and $(\Delta f)(\xi)=2 \int_{0}^{1} a(t) d t$.

For each $g \in O(H)$ we define an operator $U(g)$ :

$$
(U(g) f)(\xi)=f\left(g^{-1} \xi\right),
$$

where $f$ is an arbitrary function on $H$. The Lévy Laplacian characterizes the group $\mathscr{G}(L, \mathscr{D})$ as follows.

TheOREM 3. The group $\mathscr{G}(L, \mathscr{D})$ consists of all permutations which commute with the Lévy Laplacian $\Delta$ with domain $\operatorname{Dom}_{H}(\Delta)$.

Proof. For $g \in \mathscr{G}(L, \mathscr{D})$ and $f \in \operatorname{Dom}_{H}(\Delta)$, we shall show that

(i) $U(g) f \in \operatorname{Dom}_{H}(\Delta)$;

(ii) $\Delta U(g) f=U(g) \Delta f$.

Put

$$
a_{n}(\xi)=\frac{\partial^{2} f}{\partial \xi_{n}^{2}}(\xi), \quad \xi \in H, \quad n \geqslant 1
$$

Then, by assumption the sequence $a(\xi)=\left(a_{n}(\xi)\right)_{n=1}^{\infty}$ belongs to $\mathscr{D}$ for any $\xi \in H$. Viewing the definitions of $L$ and $\Delta$, we obtain

$$
(\Delta f)(\xi)=L(a(\xi)), \quad \xi \in H .
$$

By definition,

$$
\begin{gathered}
g a(\xi) \in \mathscr{D} \quad \text { for any } \xi \in H ; \\
L(g a(\xi))=L(a(\xi)) \quad \text { for any } \xi \in H .
\end{gathered}
$$

On the other hand, a direct calculation implies that

$$
\left(\frac{\partial^{2}}{\partial \xi_{n}^{2}} U(g) f\right)(\xi)=a_{g-1(n)}\left(g^{-1} \xi\right) .
$$

Therefore, by (3.7) we have $U(g) f \in \operatorname{Dom}_{H}(\Delta)$ and

$$
(\Delta U(g) f)(\xi)=L\left(g a\left(g^{-1} \xi\right)\right) .
$$


Hence by (3.6) and (3.8) we have

$$
(\Delta U(g) f)(\xi)=L\left(a\left(g^{-1} \xi\right)\right)=(\Delta f)\left(g^{-1} \xi\right)=(U(g) \Delta f)(\xi),
$$

as desired.

Conversely, suppose that a permutation $g \in \operatorname{Aut}(N)$ commutes with the Lévy Laplacian $\Delta$, i.e.

(iii) $\operatorname{Dom}_{H}(\Delta)$ is stable under $U(g)$;

(iv) $\Delta U(\mathrm{~g})=U(\mathrm{~g}) \Delta$.

For any $a \in \mathscr{D}$ we define a diagonal operator $A \in \boldsymbol{B}(H)$ by $A e_{n}=a_{n} e_{n}$, $n \geqslant 1$. Then $f(\xi)=(A \xi, \xi)$ belongs to $\operatorname{Dom}_{H}(\Delta)$ and $(\Delta f)(\xi) \equiv L(a)$. Therefore, as is easily seen, the assumptions (iii) and (iv) imply that $g a \in \mathscr{D}$ and that $L(g a)=L(a)$. Hence $g \in \mathscr{G}(L, \mathscr{D})$.

Q.E.D.

CoRollary 3.5. Every permutation of the Lévy group $\mathscr{G}$ commutes with the Lévy Laplacian with the domain $\operatorname{Dom}_{H}(\Delta)$.

Let $E=C^{\infty}\left(S^{1}\right)$ be the space of all $R$-valued $C^{\infty}$-functions on the circle. Equipped with countably many norms $\|\cdot\|_{p}$ :

$$
\|\xi\|_{p}^{2}=\sum_{q=0}^{p} \int_{0}^{1}\left|\xi^{(q)}(t)\right|^{2} d t, \quad \xi \in E, \quad p \geqslant 0
$$

$E$ becomes a nuclear space. We introduce the space $\operatorname{Dom}_{E}(\Delta)$ in the same manner as $\operatorname{Dom}_{H}(\Delta)$. Then the Lévy Laplacian $\Delta$ is defined on $\operatorname{Dom}_{E}(\Delta)$ by the formula (3.1).

Example 3.6. (cf. Example 3.2.) A function $f$ on $E$ is called a monomial of degree $p$ if it is of the form:

$$
f(\xi)=\left\langle a, \xi^{\otimes p}\right\rangle,
$$

where $\langle$,$\rangle denotes the canonical bilinear form on \left(E^{\otimes p}\right)^{*} \times E^{\otimes p}$. If the limit

$$
p(p-1) \lim _{N \rightarrow \infty} \frac{1}{N} \sum_{n=1}^{N}\left\langle a, e_{n} \otimes e_{n} \otimes \xi^{\otimes(p-2)}\right\rangle
$$

exists for all $\xi \in E$, then $f$ belongs to $\operatorname{Dom}_{E}(\Delta)$ and $(\Delta f)(\xi)$ is given by the above limit (3.10).

A typical monomial is given by the integral:

$$
f(\xi)=\int \cdots \int a\left(t_{1}, \cdots, t_{k}\right) \xi\left(t_{1}\right)^{p_{1}} \cdots \xi\left(t_{k}\right)^{p_{k}} d t_{1} \cdots d t_{k}
$$


where $a\left(t_{1}, \cdots, t_{k}\right)$ is an integrable function on $S^{1} \times \cdots \times S^{1}$ (k-times) and $p_{1}, p_{2}, \cdots, p_{k} \geqslant 1$. After P. Lévy [8] a linear combination of such functions is called a normal functional. In particular, the function $f(\xi)$ is called a regular functional if $p_{1}=\cdots=p_{k}=1$.

We shall determine the subgroup of $\operatorname{Aut}(N)$ which commutes with the Lévy Laplacian $\Delta$ with the domain $\operatorname{Dom}_{E}(\Delta)$. First note that the space $E$ is not stable under the orthogonal group $O(H)$. Following $H$. Yoshizawa [11] we call an orthogonal operator $g \in O(H)$ a rotation of $E$ if its restriction to $E$ induces a topological automorphism of $E$. Let $O(E, H)$ denote the group of all rotations of $E$. All of our arguments and calculations in Theorem 3 can be carried over to this case and we have the following

THEOREM 4. The group $\mathscr{G}(L, \mathscr{D}) \cap O(E, H)$ consists of all permutations which commute with the Lévy Laplacian $\Delta$ with the domain $\operatorname{Dom}_{E}(\Delta)$.

Corollary 3.7 ([6]). The group $\mathscr{G} \cap O(E, H)$ commutes with the Lévy Laplacian with the domain $\operatorname{Dom}_{E}(\Delta)$.

For the group Aut $(N) \cap O(E, H)$ we have the following result whose proof is easy and omitted.

Proposition 3.8. A permutation $g \in \operatorname{Aut}(N)$ belongs to $O(E, H)$ if and only if there exist two positive constants $\alpha$ and $\beta$ such that

(i) $\sup _{n \geqslant 1} n^{-\alpha} g(n)<\infty$;

(ii) $\inf _{n \geqslant 1} n^{-\beta} g(n)>0$.

Corollary 3.9 ([1]). Let $0=N_{0}<N_{1}<\cdots<N_{k}<\cdots$ be an increasing sequence of integers with $\lim _{k \rightarrow \infty} N_{k} / N_{k-1}=1$. Any permutation $g \in \operatorname{Aut}(N)$ which leaves every subset $\left\{N_{k-1}+1, \cdots, N_{k}\right\}$ invariant belongs to $O(E, H)$.

Proof. The condition in Proposition 3.8 is obviously satisfied if we put $\alpha=\beta=1$.

Q.E.D.

\section{§4. Further properties of the Lévy group}

In this section we shall prove the following two assertions.

Proposition 4.1. (cf. Proposition 3.1.) The Lévy group $\mathscr{G}$ is a proper subgroup of $\mathscr{G}(L, \mathscr{D})$. 
Proposition 4.2. (cf. Corollary 3.7.) $\mathscr{G}_{0}$ is not a subgroup of $O(E, H)$. In particular, the Lévy group is not a subgroup of $O(E, H)$.

Every finite subset $S \subset N$ admits a unique decomposition into a sum of disjoint segments as follows:

$$
S=\bigcup_{\ell=1}^{k}\left\{i_{\ell}+1, \cdots, i_{\ell}+j_{\ell}\right\}
$$

where $i_{\ell}+j_{\ell}<i_{\ell+1}, j_{\ell} \geqslant 1,1 \leqslant \ell \leqslant k$. Then we put

$$
\lambda(S)=\sum_{\ell=1}^{k}\left(2 i_{\ell}+j_{\ell}\right)=\sum_{\ell=1}^{k}\left(\left(i_{\ell}+1\right)+\left(i_{\ell}+j_{\ell}\right)-1\right) .
$$

Lemma 4.3. Assume that $g \in \operatorname{Aut}(N)$ satisfies the condition:

$$
\sup _{N} \frac{1}{N}\left\{\lambda\left(F_{N}^{+}(g)\right)+\lambda\left(F_{N}^{-}(g)\right)\right\}=\gamma<\infty .
$$

Then $g$ belongs to $\mathscr{G}(L, \mathscr{D})$.

Proof. Let $a \in \mathscr{D}$. Given $\varepsilon>0$ we choose $N_{0} \geqslant 1$ such that

$$
\left|\sum_{n=1}^{N}\left(a_{n}-L(a)\right)\right| \leqslant \varepsilon N \quad \text { for every } N>N_{0} \text {. }
$$

Note that

$$
\begin{aligned}
& \left|\sum_{n=p+1}^{p+q}\left(a_{n}-L(a)\right)\right| \\
& \quad \leqslant\left|\sum_{n=1}^{p+q}\left(a_{n}-L(a)\right)+\right| \sum_{n=1}^{p}\left(a_{n}-L(a)\right) \mid \leqslant \varepsilon(2 p+q),
\end{aligned}
$$

where $p \geqslant N_{0}$ and $q \geqslant 1$. If $N>N_{0}$ is sufficiently large, we have

$$
F_{N}^{+}(g) \subset\left\{N_{0}+1, N_{0}+2, \cdots\right\} .
$$

Then by (4.1) and (4.2),

$$
\begin{aligned}
& \left|\sum_{n=1}^{N}\left(a_{n}-a_{g^{-1}(n)}\right)=\right| \sum_{n \in F_{N}^{+}(g)} a_{n}-\sum_{n \in F_{N}^{-}(g)} a_{n} \mid \\
& \quad \leqslant\left|\sum_{n \in F_{N}^{+}(g)}\left(a_{n}-L(a)\right)\right|+\left|\sum_{n \in F_{N}^{-}(g)}\left(a_{n}-L(a)\right)\right| \\
& \quad \leqslant \varepsilon\left\{\lambda\left(F_{N}^{+}(g)\right)+\lambda\left(F_{N}^{-}(g)\right)\right\} .
\end{aligned}
$$

Therefore,

$$
\frac{1}{N} \sum_{n=1}^{N}\left(a_{n}-a_{g-1(n)}\right) \leqslant \varepsilon \gamma
$$


for every sufficiently large $N>N_{0}$. This implies that $g a$ belongs to $\mathscr{D}$ and that $L(g a)=L(a)$.

Q.E.D.

Proof of Proposition 4.1. Fix an increasing sequence $0=N_{0}<N_{1}<$ $N_{2}<\cdots$ such that $N_{k}-N_{k-1}=2 m_{k}, m_{k} \in N$. Define $g \in \operatorname{Aut}(N)$ as product of disjoint transpositions as follows:

$$
g=\prod_{k=1}^{\infty} \prod_{j=1}^{m_{k}}\left(N_{k-1}+j, N_{k-1}+m_{k}+j\right) .
$$

It can be easily shown that if $N_{k-1}<N \leqslant N_{k}$,

$$
\lambda\left(F_{N}^{+}(g)\right)=N_{k-1}+N, \quad \lambda\left(F_{N}^{-}(g)\right)=N_{k}+N .
$$

Hence

$$
\frac{1}{N}\left\{\lambda\left(F_{N}^{+}(g)\right)+\lambda\left(F_{N}^{-}(g)\right)\right\}=2+\frac{1}{N}\left(N_{k-1}+N_{k}\right) \leqslant 3+\frac{N_{k}}{N_{k-1}} .
$$

Thus, if $\sup _{k \geqslant 2} N_{k} / N_{k-1}<\infty$, by Lemma 4.3 we see that $g \in \mathscr{G}(L, \mathscr{D})$. While, it is easy to see that

$$
F(g)=\limsup _{N \rightarrow \infty} \frac{1}{N}\left|F_{N}(g)\right|=2 \limsup _{k \rightarrow \infty} \frac{N_{k}-N_{k-1}}{N_{k}+N_{k-1}} .
$$

Therefore, there exists a permutation $g \in \mathscr{D}(L, \mathscr{D})$ with $F(g)>0$. This completes the proof.

Q.E.D.

Proof of Proposition 4.2. Let $2=n_{1}<n_{2}<\cdots$ be an increasing sequence of prime numbers. We put

$$
g=\prod_{k=1}^{\infty}\left(n_{k} 2^{n_{k}}\right) \in \operatorname{Aut}(N) .
$$

It follows from the prime number theorem that $\delta(\operatorname{supp} g)=0$, namely, $g \in \mathscr{G}_{0}$. While, for any $\alpha>0$, we have

$$
\sup _{n \geqslant 1} n^{-\alpha} g(n) \geqslant \sup _{k \geqslant 1} n_{k}^{-\alpha} 2^{n_{k}}=\infty .
$$

Therefore $g$ does not belong to $O(E, H)$ by Proposition 3.8. $\quad$ Q.E.D.

\section{REFERENCES}

[1] T. Hida, Brownian motion, Applications of Mathematics 11, Springer-Verlag, 1980.

[2] — B Brownian motion and its functionals, Ricerche Mat., 34 (1985), 183-222.

[ 3 ] — - Analysis of Brownian functionals, Lecture Notes, IMA, University of Minnesota, 1986. 
[4] T. Hida, I. Kubo, H. Nomoto and H. Yoshizawa, On projective invariance of Brownian motion, Publ. RIMS Kyoto Univ., ser. A, 4 (1969), 595-609.

[5] T. Hida, K.-S. Lee and S.-S. Lee, Conformal invariance of white noise, Nagoya Math. J., 98 (1985), 87-98.

[6 ] T. Hida and K. Saitô, White noise analysis and the Lévy Laplacian, to appear in Proc. BiBoS IV (1986).

[ 7 ] I. Kubo and S. Takenaka, Calculus on Gaussian white noise IV, Proc. Japan Acad., 58 ser. A (1982), 186-189.

[8] P. Lévy, Problèmes concrets d'analyse fonctionnelle, Part 3, Gauthier-Villars, Paris, 1951.

[ 9 ] K. Saitô, Itô's formula and Lévy Laplacian, Nagoya Math. J., 108 (1987), 67-76.

[10] Y. Yamasaki, Measures on infinite dimensional spaces, Series in Pure Mathematics Vol. 5, World Scientific, 1985.

[11] H. Yoshizawa, Rotation group of Hilbert space and its application to Brownian motion, Proceedings of the International Conference on Functional Analysis and Related Topics, Tokyo, 1969, 414-423.

[12] A. Zygmund, Trigonometric series I, Chapter III, Oxford Univ. Press 1959.

Department of Mathematics

Faculty of Science

Nagoya University

Chikusa-ku, Nagoya, 464 Japan 